\title{
Bringing Standardized Processes to Atom-Probe Tomography - Part 1: Establishing Standardized Terminology
}

\author{
E.A. Marquis (Chair), ${ }^{*}$ I.M. Anderson, ${ }^{* *}$ F. Danoix, ${ }^{* * *}$ R.G. Forbes,${ }^{* *}{ }^{* \dagger}$ B. Gault,${ }^{\dagger}$ T.F. Kelly,$^{\dagger \dagger}$ \\ M.K. Miller, ${ }^{\dagger \dagger}$ M.P. Moody, ${ }^{\dagger}$ and F. Vurpillot***
}

* Department of Materials Science \& Engineering, University of Michigan, Ann Arbor, MI 48109

** Surface \& Microanalysis Science Division, National Institute for Standards \& Technology, Gaithersburg, MD 20899

*** Groupe de Physique des Matériaux, University of Rouen, F-76801 St Etienne du Rouvray, France

$*^{* * \dagger}$ Advanced Technology Institute, University of Surrey, Guildford GU2 5XH, United Kingdom

${ }^{\dagger}$ Australian Center for Microscopy \& Microanalysis, University of Sydney, Sydney, NSW 2006 Australia

${ }^{\dagger \dagger}$ Cameca Instruments Inc., Madison, WI 53711

It广 Materials Science \& Technology Division, Oak Ridge National Laboratory, Oak Ridge, TN 37831

There is rarely a unique answer to a materials analysis question. Even with analysis by an individual technique, the approach used to address the question can shape the answer, and there are often several valid approaches. The results depend upon the specifics of the approach, which can include such variables as the specimen preparation, analysis methodology, data acquisition and analysis tools, and error analysis. This approach-specific aspect of scientific analyses can undermine confidence in analytical methods when the results of various approaches conflict, and can cause problems from wasted resources to unacceptable materials failures. Establishing consistent methods that deliver reproducible results with quantifiable errors can often improve the reliability of the method and the interpretation of multiple sets of data. In the rapidly maturing field of atom-probe tomography (APT), such standard approaches are needed.

While an obvious concept, standardization of method requires careful consideration of the entire APT field and its applications. Following preliminary efforts [1], the International Field Emission Society (IFES) has elected a Standards Committee, whose membership is listed above, which is tasked to determine the needed steps to establish APT as an accepted metrology technique. Specific tasks include developing protocols or standards for: (i) terminology and nomenclature, (ii) protocols for test methodologies, (iii) protocols for metrology and instrumentation, (iv) specifications for standard reference materials, (v) protocols for modeling and simulations, and (vi) science-based health, safety, and environmental practices.

The Standards Committee is currently working on defining terminology related to APT with the goal being the inclusion of terms into a document published by the International Organization for Standards (ISO). Many terms common to other techniques and disciplines have already been defined [2], and will be considered for adoption in the context of atom-probe tomography. A preliminary list of terms can be found in Table 1 below.

[1] R.M. Ulfig, T.F. Kelly, and B. Gault, Microsc. Microanal. 15 (Suppl. 2) (2009) 260.

[2] Surface Chemical Analysis - Vocabulary - Part 1: General Terms and Terms for the Spectroscopies, ISO/CD 18115-1, International Organization for Standards, 2008. 
[3] Research was supported by Oak Ridge National Laboratory's Shared Research Equipment (SHaRE) User Facility, which is sponsored by the Office of Basic Energy Sciences, US Department of Energy (MKM).

TABLE 1. : Initial list of terms for which a standard terminology is being developed in conjunction with [2].

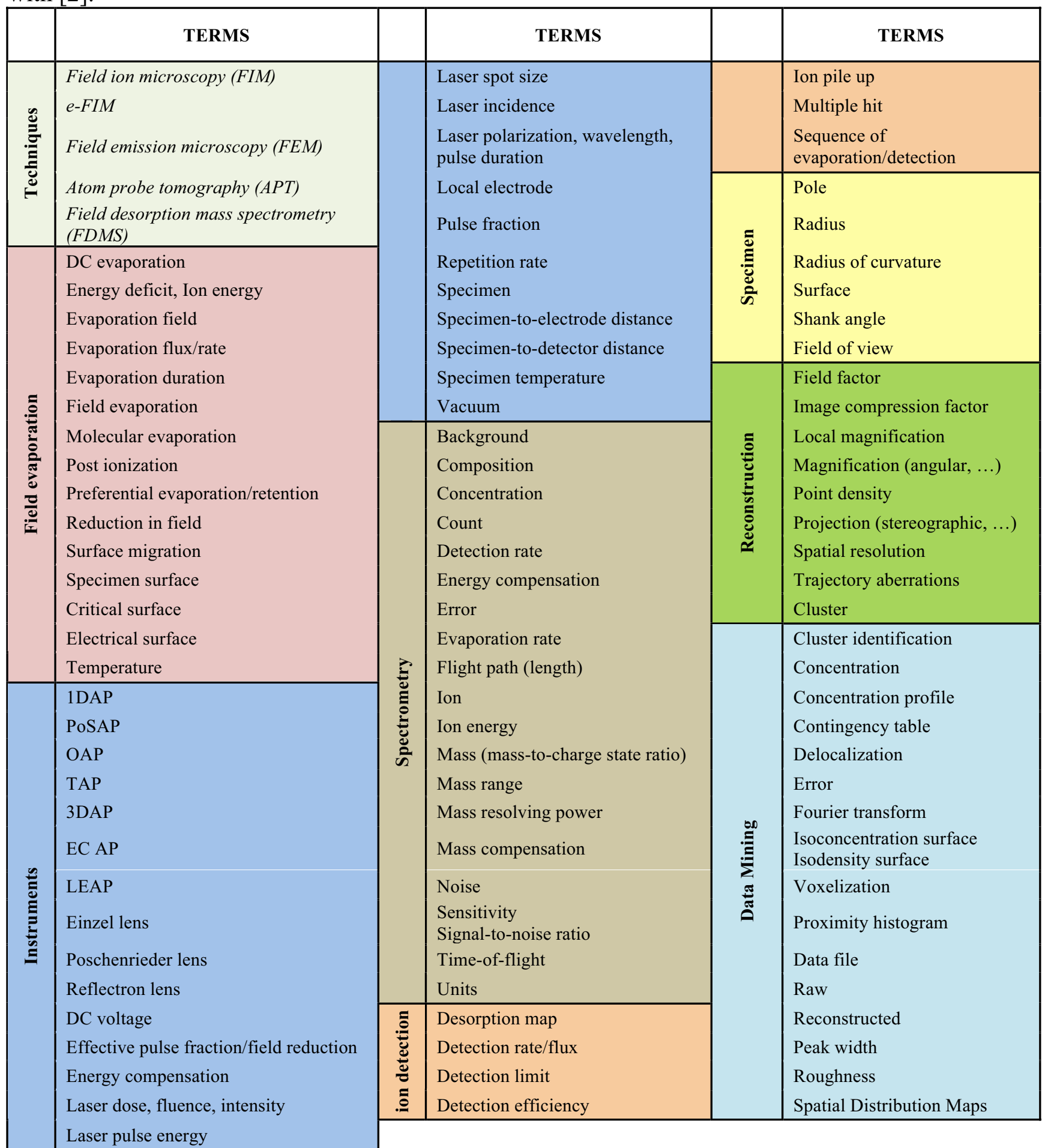

